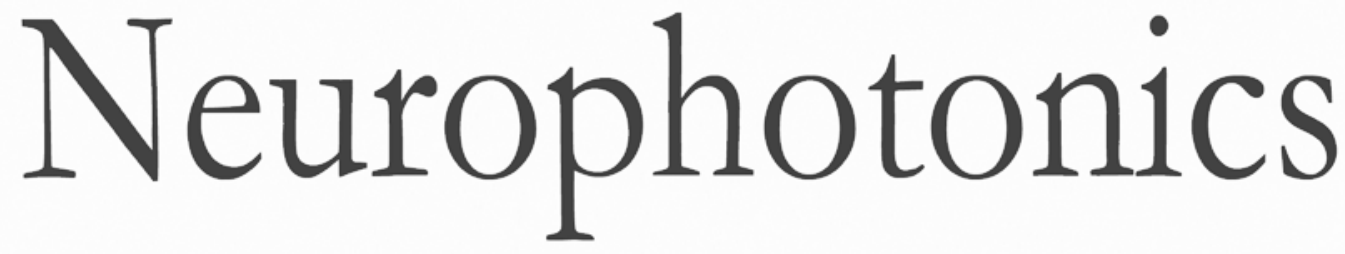

\title{
Time-domain near-infrared spectroscopy in acute ischemic stroke patients
}

Giacomo Giacalone

Marta Zanoletti

Rebecca Re

Bruno Germinario

Davide Contini

Lorenzo Spinelli

Alessandro Torricelli

Luisa Roveri 


\title{
Time-domain near-infrared spectroscopy in acute ischemic stroke patients
}

\author{
Giacomo Giacalone, ${ }^{\mathrm{a}, \mathrm{b}, *}$ Marta Zanoletti, ${ }^{\mathrm{c}}$ Rebecca Re, ${ }^{\mathrm{c}}$ Bruno Germinario, ${ }^{\mathrm{a}}$ Davide Contini, ${ }^{\mathrm{c}}$ \\ Lorenzo Spinelli, ${ }^{d}$ Alessandro Torricelli, ${ }^{c, d}$ and Luisa Roveri ${ }^{\mathrm{a}, \mathrm{b}, *}$ \\ ${ }^{a}$ San Raffaele Scientific Institute, Neurology Department, Via Olgettina, Milan, Italy \\ 'San Raffaele "Vita-Salute" University, Via Olgettina, Milan, Italy \\ 'Politecnico di Milano, Dipartimento di Fisica, Piazza Leonardo da Vinci, Milan, Italy \\ distituto di Fotonica e Nanotecnologie, Consiglio Nazionale delle Ricerche, Piazza Leonardo da Vinci, Milan, Italy
}

\begin{abstract}
Large vessel occlusion (LVO) stroke might cause different degrees of hemodynamic impairment that affects microcirculation and contributes to metabolic derangement. Time-domain near-infrared spectroscopy (TD-NIRS) estimates the oxygenation of microcirculation of cerebral outer layers. We measure hemoglobin species and tissue oxygen saturation $\left(\mathrm{StO}_{2}\right)$ of anterior circulation stroke patients, classified as LVO or lacunar, and assess the differences compared with controls and according to LVO recanalization status. Fiducial markers categorize the brain region below each TD-NIRS probe as ischemic or nonstroke areas. The study includes 47 consecutive acute ischemic stroke patients and 35 controls. The ischemic area has significantly higher deoxy-hemoglobin $(\mathrm{HbR})$ and total hemoglobin $(\mathrm{HbT})$ compared with controls in both recanalized and nonrecanalized patients but lower $\mathrm{StO}_{2}$ only in recanalized patients. Recanalized patients have significantly lower mean $\mathrm{StO}_{2}$ in the ipsilateral hemisphere compared with nonrecanalized patients. This is the first study to report TD-NIRS measurements in acute ischemic stroke patients. TD-NIRS is able to detect significant differences in hemoglobin species in LVO stroke compared with controls and according to recanalization status. This preliminary data might suggest that $\mathrm{StO}_{2}$ can serve as a surrogate functional marker of the metabolic activity of rescued brain tissue. ( ) The Authors. Published by SPIE under a Creative Commons Attribution 4.0 Unported License. Distribution or reproduction of this work in whole or in part requires full attribution of the original publication, including its DOI. [DOI: 10.1117/1.NPh.6.1.015003]
\end{abstract}

Keywords: near-infrared spectroscopy; acute ischemic stroke; near-infrared spectroscopy; large vessel occlusion; revascularization; oximetry.

Paper 18049R received Aug. 29, 2018; accepted for publication Nov. 28, 2018; published online Feb. 7, 2019.

\section{Introduction}

Acute ischemic stroke is a highly dynamic condition. Indeed, large vessels occlusion (LVO) might cause different degrees of hemodynamic impairment that result in complex interplay between LVO, collateral status, and clinical symptomatology. The study of microcirculation, which is the site of cerebral autoregulation and neurovascular coupling, might be a valuable strategy to improve our insight in this field. The concept of the neurovascular unit is one of the key elements of stroke pathophysiology. It has been shown that capillary flow patterns undergo profound changes in cerebral ischemia and affect local oxygen delivery. ${ }^{1}$ Modifications in the capillary bed contribute to the neurovascular dysfunction and to the metabolic derangement in the ischemic tissue, where tissue survival depends on oxygen extraction to compensate for the reduced perfusion. Other factors such as involvement of small cerebral veins and the role of the rete vasorum remain undefined. ${ }^{2}$ In addition, secondary changes may develop in ipsilateral hemisphere outside the ischemic area and in the contralateral hemisphere as well. The alteration of regional cerebral blood flow and metabolism in the contralateral hemisphere has been reported. ${ }^{3-5}$ Near-infrared spectroscopy (NIRS) is an optical technique that provides information about oxygenation in the microcirculation of cerebral outer layers. ${ }^{6,7}$ Furthermore, it is

*Address all correspondence to Giacomo Giacalone, E-mail: giacalone .giacomo@ hsr.it; Luisa Roveri, E-mail: roveri.luisa@hsr.it. a noninvasive, safe, and portable methodology that enables real-time monitoring at the bedside of the patient. ${ }^{6,7}$ Technical advances have overcome some limitations of the previous NIRS systems. Indeed, the contamination from the extra-cerebral layers and the possibility to retrieve only relative changes with respect to a baseline were the most limiting drawbacks of the continuous-wave NIRS (CW-NIRS) technique. ${ }^{8}$ Advanced optical techniques, such as time-domain NIRS (TD-NIRS) and frequency-domain (FD)-NIRS, allow to recover the absolute concentrations of hemoglobin species and to better separate the contribution of the extra-cerebral layers from the brain cortical ones. ${ }^{6}$ In particular, in TD-NIRS with single source-detector pair, the effect of extra-cerebral layers can be marginal even if fitting with homogeneous model is used. ${ }^{9}$ Therefore, the estimation of brain optical properties is less dependent on the superficial layers. Of course, the use of a multidistance approach and of a priori information on the head anatomy could further improve the accuracy of the estimate, at the cost of increased complexity of the approach. A recent study reported TD-NIRS measurements of cerebral deoxyhemoglobin (HbR), oxyhemoglobin $(\mathrm{HbO})$, total hemoglobin $(\mathrm{HbT}=\mathrm{HbR}+\mathrm{HbO})$, and tissue oxygen saturation $\left(\mathrm{StO}_{2}=\mathrm{HbO} / \mathrm{HbT}\right)$ values of healthy subjects, demonstrating the reproducibility of results. ${ }^{10}$ The study of the cerebral microcirculation by means of cerebral timedomain near infrared spectroscopy (TD-NIRS) could inform about the effect of LVO. The vast majority of studies in ischemic stroke patients have used CW-NIRS, ${ }^{11-17}$ whereas only few were performed with FD-NIRS. ${ }^{18,19}$ In addition, in all but one 
previous studies, ${ }^{18}$ recordings were limited to the forehead of the subjects, ${ }^{11-16,19}$ irrespective of the site of ischemic area.

The aim of this prospective study was to evaluate the concentration of hemoglobin species and $\mathrm{StO}_{2}$ as measured by TD-NIRS in acute ischemic stroke patients, examining both ischemic and nonstroke brain areas. In addition, we assessed the presence of differences compared with control subjects and according to LVO recanalization status.

\section{Methods}

The study received approval by the Institutional Ethical Committee and by the Ministry of Health and was conducted in compliance with the Declaration of Helsinki. An informed consent was obtained from both patients and controls. We prospectively enrolled consecutive acute ischemic stroke patients with the following inclusion criteria: (a) involvement of the anterior circulation, (b) able to perform TD-NIRS measurements within $24 \mathrm{~h}$ from stroke onset, and (c) absence of respiratory insufficiency or need of $\mathrm{O}_{2}$-therapy at the time of the measurements. Patients underwent clinical and neuroimaging evaluation as per standard of care for acute stroke patients including National Institute of Health Stroke Scale (NIHSS) assessment, brain computed tomography (CT) or magnetic resonance (MR), CT or MR angiography, digital subtraction angiography, and transcranial Doppler ultrasonography. At baseline, stroke subtypes were classified according to the Oxfordshire Community Stroke Project Classification (OCSP) ${ }^{20}$ in partial/total anterior circulation syndrome (PACS/TACS) and lacunar syndrome (LACS). Neurovascular imaging performed at baseline assessed LVO, and follow-up imaging assessed recanalization status.

Patients with PACS/TACS were divided into two groups: (i) recanalized LVO including patients with recanalization either spontaneous or subsequent to treatment [recombinant tissue plasminogen activator (rtPA) and/or mechanical thrombectomy] and (ii) nonrecanalized LVO.

To avoid any delay in treatment, for patients eligible for the recanalization procedure (rtPA and/or thrombectomy), TD-NIRS recording was performed afterward. Baseline OCSP clinical syndromes classification was confirmed as OCSP infarcts classification by follow-up neuroimaging. ${ }^{20}$ Subjects older than 65 years were selected as a control group from a cohort of healthy volunteers described in a previous study. ${ }^{9}$

TD-NIRS measurements were performed in the Stroke Unit, at the bedside of the patient, in condition of dimmed light, with the patient resting with an angle-of-bed inclination of $30 \mathrm{deg}$. Arterial blood pressure and peripheral pulse oximetry $\left(\mathrm{SpO}_{2}\right)$ were acquired during each recording session.

TD-NIRS measurements were obtained in both controls and patients by placing a pair of illuminating and collecting optical fibers (i.e., an optical probe) in selected standard positions of frontal, central, and parietal brain regions of each hemisphere using an EEG cap (g.EEGcap, g.tec medical engineering $\mathrm{GmbH}$, Austria) according to the 10-10 international positioning system (F3-F5, C3-C1, P3-P5 for the left hemisphere and F4-F6, C4-C2, P4-P6 for the right hemisphere). For stroke patients, additional positions of interest for optical probes were identified at baseline according to the OCSP classification ${ }^{20}$ combined with neuroimaging features [ischemic signs (ASPECTS) on baseline CT scan $;{ }^{21}$ site of large vessel involvement] and with arterial territory maps of human brain $^{22}$ (Fig. 1). To classify the probed brain areas, we designed a cap equipped with fiducial markers on the same positions of the EEG cap used for TDNIRS measurements (Fig. 2). By projecting orthogonal lines from the fiducial markers to the brain in 3-D reconstructed images $\left(\mathrm{XERO}^{\circledR}\right.$ Viewer-Agfa HealthCare), we could classify the brain area underlying each position of the optical probe as ischemic or nonstroke area according to follow-up neuroimaging (Fig. 2). We discarded recordings with optical probe falling across two different areas (stroke and nonstroke) or on atrophic or past-injured tissue. Recordings were obtained from positions of interest on the affected hemisphere and the matched area on the contralateral hemisphere. The recording session consisted of three sets of measurements for each position of interest repeated at two time points: within $24 \mathrm{~h}$ of stroke symptoms onset (first time point) and after $24 \mathrm{~h}$ from the first time point up to 5 days (second time point).

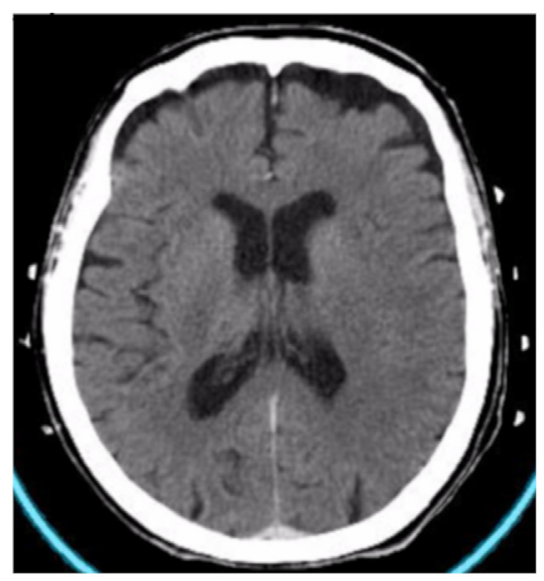

(a)

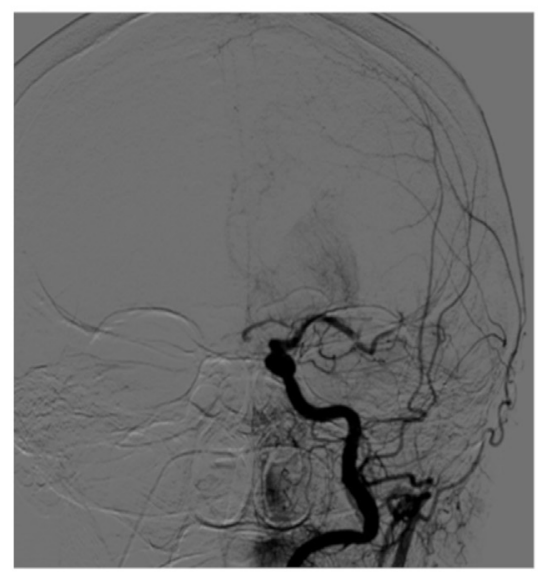

(b)

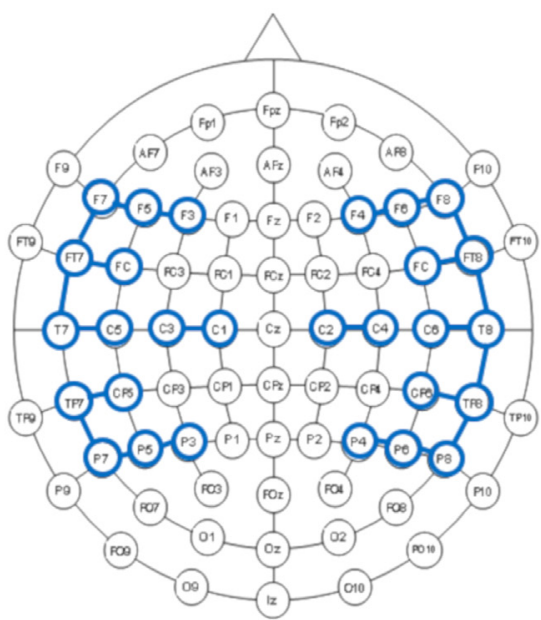

(c)

Fig. 1 Selection of positions for TD-NIRS measurement. Example of positions' selection for TD-NIRS measurement in a patient with TACS. (a) baseline CT-scan showing indirect ischemic signs in the left hemisphere (white extracranial dots are the fiducial markers), (b) digital subtraction angiography showing occlusion of left middle cerebral artery (distal M1 tract), and (c) selected positions for TD-NIRS measurement (in light blue) on the 10-10 international positioning system. 


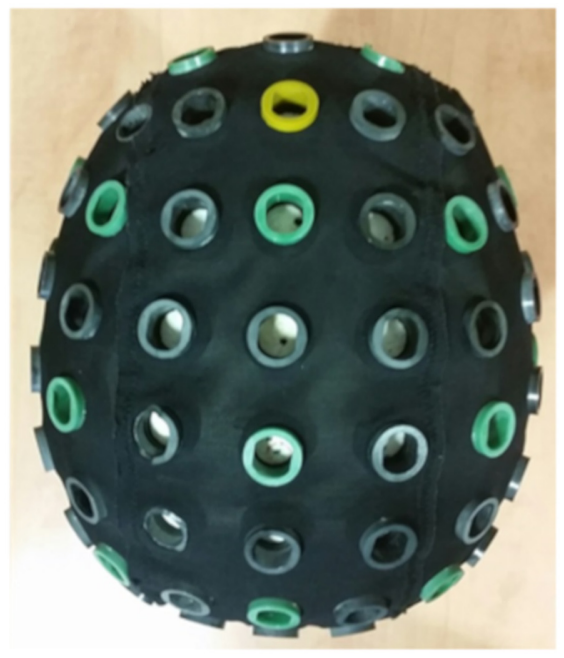

(a)

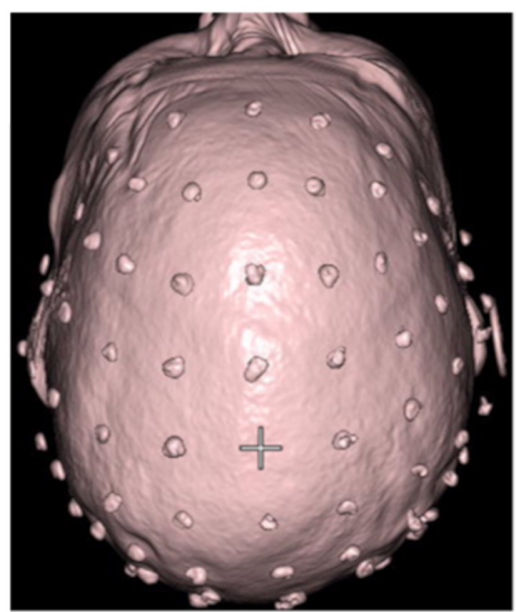

(c)

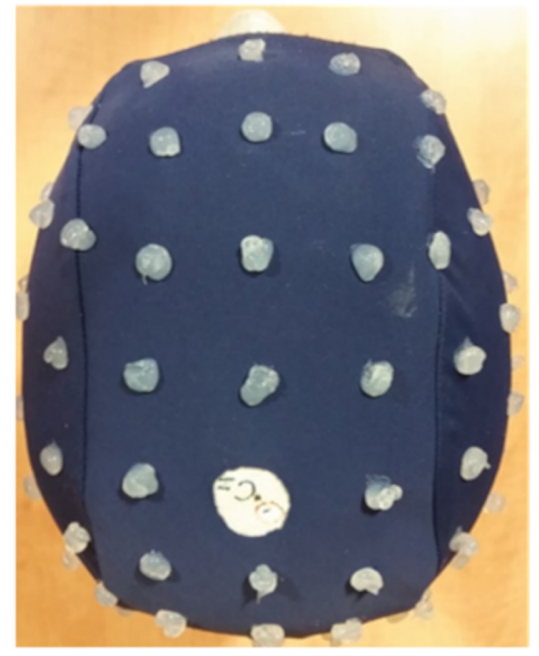

(b)

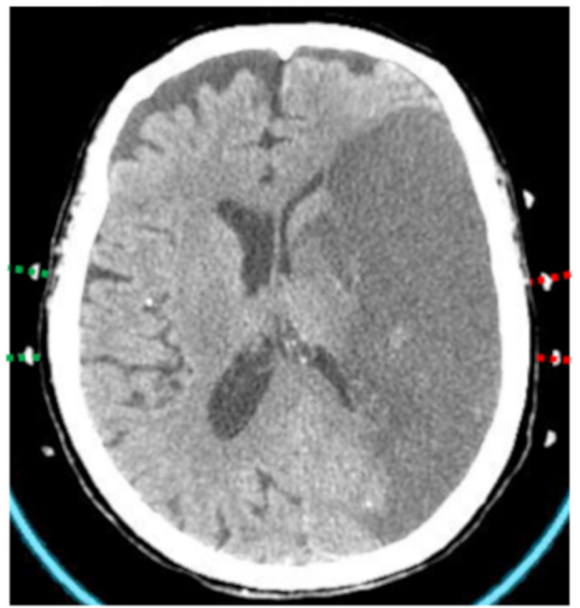

(d)

Fig. 2 Matching of TD-NIRS positions with characteristics of probed brain region. Example of matching of TD-NIRS measurements with the characteristic of brain area underlying illuminating and collecting optical fibers. (a) EEG cap (10-10 international positioning system) used for TD-NIRS measurements, (b) cap equipped with fiducial markers on the same positions of the EEG cap, for neuroimaging investigation, (c) 3-D recostructed images of head CT scan, (d) projection of orthogonal lines from fiducial markers to the scalp in correspondence with the ischemic lesion and specular contralateral nonstroke area (respectively dashed red and green lines).

\subsection{Instrumentation and Data Acquisition}

TD-NIRS data were acquired by means of a device designed and developed at the Department of Physics of Politecnico di Milano. ${ }^{9}$ The light sources are three pulsed diode lasers working at 690, 785, and $830 \mathrm{~nm}$, respectively (mod. LDH series, PicoQuant $\mathrm{GmbH}$, Germany) and at a $80-\mathrm{MHz}$ repetition rate. Laser pulses are injected into the tissue by a multimode-graded index optical fibers $(100 / 140 \mu \mathrm{m}$ core/cladding diameter) paying attention in maintaining the power density below the maximum permissible exposure of $2 \mathrm{~mW} / \mathrm{mm}^{2}$ thanks to a spacer placed at the tip of the probe. Photons are collected from the tissue in reflectance mode by means of a custommade three furcate fiber bundle made of graded index plastic fibers (1-mm core diameter, 0.29 NA; mod. OM-GIGA POF, Luceat Srl, Italy) and then detected by three hybrid photomultipliers (mod. HPM-100-50, Becker-Hickl, GmbH, Germany), each dedicated to a specific wavelength using bandpass filters centered at 690, 785, and $830 \mathrm{~nm}$ (mod. Hard Coated OD4, $10 \mathrm{~nm}$ Bandpass Filters, Edmund Optics GmbH, Germany). Finally, three time-correlated single photon counting boards acquire the distributions of photon time of flight that is the TD-NIRS signal or curves.

The instrument response function (IRF), obtained by facing the injection fiber with the collection fiber, features a full width at half maximum $(\mathrm{FWHM})<200 \mathrm{ps}$.

\subsection{Data Analysis}

Quality control criteria described in details in a previous study ${ }^{10}$ were applied to data of stroke patients and control subjects. They included the number of detected photons, the minimization of the least square error between experimental data and theoretical model of photon diffusion $\left(\chi^{2}\right)$, the reproducibility of measurement on a calibrated phantom, and the difference between the 
temporal position of TD-NIRS experimental curves and the IRF $\left(\Delta t_{\text {TDNIRS-IRF }}\right)$.

At each recording session, the values of $\mathrm{HbR}, \mathrm{HbO}, \mathrm{HbT}$, and $\mathrm{StO}_{2}$ of every single position were averaged across the three sets of measurement. Final data for analysis consisted of grand averages of positions belonging to the ischemic area, to the ipsilateral nonstroke area, and to the contralateral nonstroke area.
Clinical and demographic variables were compared using a two-sided independent sample $t$-test for continuous normal variables, $\chi^{2}$ for categorical variables, and Mann-Whitney for nonparametric variables. Group comparisons for hemoglobin species $(\mathrm{HbR}, \mathrm{HbO}, \mathrm{HbT})$ and $\mathrm{StO}_{2}$ were performed with a generalized linear mixed model using patient's categories, brain regions, and time points as fixed effects and subjects as random effects. The correction for multiple testing was performed with

Table 1 Characteristics of stroke patients.

\begin{tabular}{|c|c|c|c|c|}
\hline & $\operatorname{LACS}(n=5)$ & Recanalized LVO $(n=18)$ & Nonrecanalized LVO $(n=18)$ & $P^{\mathrm{d}}$ \\
\hline Age $(y)^{a}$ & $75.4 \pm 5.4$ & $76 \pm 9.6$ & $76.3 \pm 13.4$ & 0.94 \\
\hline Gender (M/F) & $1 / 4$ & $3 / 15$ & $5 / 13$ & 0.42 \\
\hline NIHSS at arrival ${ }^{\mathrm{b}}$ & $4(4$ to 5$)$ & $16(12$ to 19.75$)$ & 18 (15 to 22$)$ & 0.034 \\
\hline NIHSS first time point ${ }^{\mathrm{b}}$ & $4(3 \text { to } 6)^{c}$ & $9.5(4 \text { to } 13)^{c}$ & $18(16 \text { to } 21)^{c}$ & 0.003 \\
\hline NIHSS second time point ${ }^{\mathrm{b}}$ & $2(0 \text { to } 2)^{c}$ & $4.5(1 \text { to } 9.25)^{c}$ & $17(12 \text { to } 19)^{c}$ & 0.001 \\
\hline $\mathrm{mRS}$ at 3 months $^{\mathrm{b}}$ & $1(0$ to 2$)$ & $3(0.5$ to 3$)$ & 5 (4 to 5 ) & $<0.001$ \\
\hline ASPECTS $^{b}$ & $10(10$ to 10$)$ & $10(8$ to 10$)$ & 5 (3 to 6$)$ & $<0.001$ \\
\hline \multicolumn{5}{|l|}{ OCSP } \\
\hline $\mathrm{LACl}$ & 5 & 0 & 0 & 1 \\
\hline $\mathrm{PACl}$ & 0 & 16 & 2 & $<0.001$ \\
\hline $\mathrm{TACl}$ & 0 & 2 & 16 & $<0.001$ \\
\hline \multicolumn{5}{|l|}{ Treatment } \\
\hline rTPA & 1 & 8 & 2 & 0.03 \\
\hline IAT & 0 & 9 & 3 & 0.03 \\
\hline Onset-to-first time point $(h)^{a}$ & $21 \pm 4^{\mathrm{c}}$ & $15 \pm 8^{c}$ & $19 \pm 5^{c}$ & 0.25 \\
\hline Onset-to-second time point $(h)^{a}$ & $67 \pm 27$ & $84 \pm 25$ & $86 \pm 25$ & 0.8 \\
\hline First to second time point $(h)^{a}$ & $64 \pm 14^{c}$ & $58 \pm 24^{c}$ & $50 \pm 16^{c}$ & 0.32 \\
\hline \multicolumn{5}{|l|}{ Mean ABP $(\mathrm{mmHg})$} \\
\hline First time point ${ }^{\mathrm{a}}$ & $92.3 \pm 7.7^{c}$ & $94.5 \pm 16.0^{c}$ & $98.2 \pm 14.1^{\mathrm{c}}$ & 0.99 \\
\hline Second time point ${ }^{\mathrm{a}}$ & $93.3 \pm 15.4$ & $86.5 \pm 24.9$ & $97.3 \pm 10.0$ & 0.68 \\
\hline \multicolumn{5}{|l|}{ Heart rate (beats/min) } \\
\hline First time point ${ }^{\mathrm{a}}$ & $74 \pm 15.9^{c}$ & $75.6 \pm 14.8^{c}$ & $80 \pm 13.4^{c}$ & 0.83 \\
\hline Second time point ${ }^{\mathrm{a}}$ & $69.9 \pm 8.2$ & $74.6 \pm 14.1$ & $81.8 \pm 9.8$ & 0.24 \\
\hline \multicolumn{5}{|l|}{$\mathrm{SpO}_{2}(\%)$} \\
\hline First time point ${ }^{a}$ & $96.3 \pm 1.1^{\mathrm{c}}$ & $96.6 \pm 2.0^{c}$ & $96.5 \pm 2.5^{c}$ & 0.69 \\
\hline Second time point ${ }^{\mathrm{a}}$ & $96.8 \pm 0.8$ & $95.5 \pm 1.6$ & $95.5 \pm 1.6$ & 0.99 \\
\hline \multicolumn{5}{|l|}{$\mathrm{Hb}(\mathrm{g} / \mathrm{dL})$} \\
\hline First time point ${ }^{\mathrm{a}}$ & $14.5 \pm 0.4^{\mathrm{c}}$ & $12.6 \pm 2.3^{\mathrm{c}}$ & $13.4 \pm 2.4^{\mathrm{c}}$ & 0.78 \\
\hline Second time point ${ }^{a}$ & $14.2 \pm 0.6$ & $11.9 \pm 2.0$ & $13.1 \pm 2.1$ & 0.16 \\
\hline
\end{tabular}

${ }^{a}$ Mean \pm standard deviation;

${ }^{b}$ Median (interquartile range);

'NIHSS calculated on patients with available TD-NIRS at both time points ( $n=10$ recanalized, $n=11$ nonrecanalized, $n=3$ LACS);

${ }^{d} p$-value for the comparison between recanalized LVO and nonrecanalized LVO patients. ASPECTS, Alberta Stroke Program Early CT Score; OCSP, neuroradiological Oxfordshire Community Stroke Project Classification; LACI, lacunar anterior circulation infarct; PACl, partial anterior circulation infarct; TACI, total anterior circulation infarct; rTPA, recombinant-tissue plasminogen activator; IAT, intra-arterial thrombectomy. 
the FDR method. To explore the association of neurological outcome with hemoglobin species and $\mathrm{StO}_{2}$, we analyzed: (1) the improvement in stroke severity, defined as (baseline NIHSS)—(discharge NIHSS) $\geq 0$; (2) the functional outcome measured by modified Rankin scale (mRS) at 90 days after stroke dichotomized for statistical analysis in a good functional outcome $m R S \leq 3$ and poor functional outcome $m R S>3$.

Data analysis was performed with lmer package of Rstudio version 1.1.453 (ㄷ)2009-2018 RStudio, Inc.).

\section{Results}

The patient cohort included 47 consecutive acute ischemic stroke patients. We recorded a mean of 14 positions per patient (7 per hemisphere) (range 6 to 24). Quality controls yielded to the exclusion of $34.1 \%$ of the measurements $\left(15.5 \%\right.$ for $\chi^{2}$, $7.4 \%$ phantom quality, $2.2 \%$ for $\Delta t_{\text {TDNIRS-IRF }}, 9 \%$ for more than one quality control) leaving for final analysis, 24 patients with TD-NIRS measurements at both first and second time points and 41 patients with TD-NIRS measurement at the second time point. According to the OCSP classification, ${ }^{20} 36$ stroke patients were classified as PACS/TACS (18 patients as recanalized LVO and 18 patients as nonrecanalized LVO), and five patients as LACS. Baseline demographics, clinical features, mean peripheral blood hemoglobin concentration, arterial blood pressure, heart rate, $\mathrm{SpO}_{2}$, and time-to-TD-NIRS measurements were balanced among LVO stroke patients (Table 1). Stroke severity measured with NIHSS was stable between the two time points in both recanalized LVO [median (IQR), respectively, 9.5 (4 to 13 ) versus 4.5 (1 to 9.25 ), $p=0.22$ ] and nonrecanalized LVO patients [median (IQR), respectively, 18 (16 to 21 ) versus 17 (12 to 19$), p=0.36$ )] with available measurements at both time points. The control group included 35 healthy subjects with mean age of $74.1 \pm 5.4$ years, comparable with ischemic stroke patients [76.0 $\pm 10.9, p=0.31]$. The mean $\mathrm{SpO}_{2}( \pm \mathrm{SD})$ in controls was $97 \%( \pm 1.5)$.

The median within-area coefficient of variation (standard deviation/mean) of the probed positions was $<15 \%$ for $\mathrm{HbR}$, $\mathrm{HbO}, \mathrm{HbT}$ (IQR: first quartile $<10 \%$, second quartile $<20 \%$ ) and $<5 \%$ for $\mathrm{StO}_{2}$ (IQR: first quartile $<3.5 \%$, second quartile $<7 \%$.). In Fig. 3, we report an example of a fit of the distributions of photon time of flight (DTOF) at $690 \mathrm{~nm}$ obtained in a single patient from a position within the nonstroke region of the contralateral hemisphere and from a position within the ischemic area.

Patients with LVO stroke, regardless of recanalization status, had a higher concentration of $\mathrm{HbR}$ and lower $\mathrm{StO}_{2}$ values in ipsilateral and contralateral hemispheres compared with controls at both time points (ipsilateral hemisphere versus controls: first time point $\mathrm{HbR} p=1.4 \times 10^{-4}, \mathrm{StO}_{2} p=0.004$; second time point $\mathrm{HbR} p=8.2 \times 10^{-5}, \mathrm{StO}_{2} p=2.3 \times 10^{-5}$; contralateral hemisphere versus controls, first time point $\mathrm{HbR} p=0.002$, $\mathrm{StO}_{2} p=0.002$; second time point $\mathrm{HbR} p=1.4 \times 10^{-4}$, $\mathrm{StO}_{2} p=2.9 \times 10^{-6}$ ) (Fig. 4 and Table 2).

Within $24 \mathrm{~h}$ from symptoms onset, recanalized LVO patients had increased $\mathrm{HbR}$ and decreased $\mathrm{StO}_{2}$ in the ipsilateral and the contralateral hemisphere compared with controls (respectively, $\mathrm{HbR} p=5.9 \times 10^{-3}, p=0.03 ; \mathrm{StO}_{2} p=6.5 \times 10^{-4}$, $\left.p=7.9 \times 10^{-4}\right)($ Fig. 4 and Table 3). The ipsilateral nonstroke area had decreased $\mathrm{StO}_{2}$ compared with controls $(p=$ $\left.2.0 \times 10^{-4}\right)($ Fig. 4 and Table 3$)$. The ischemic area of recanalized LVO patients had increased $\operatorname{HbR}\left(p=8.2 \times 10^{-4}\right)$, increased $\mathrm{HbT}(p=0.03)$, and decreased $\mathrm{StO}_{2}(p=0.015)$

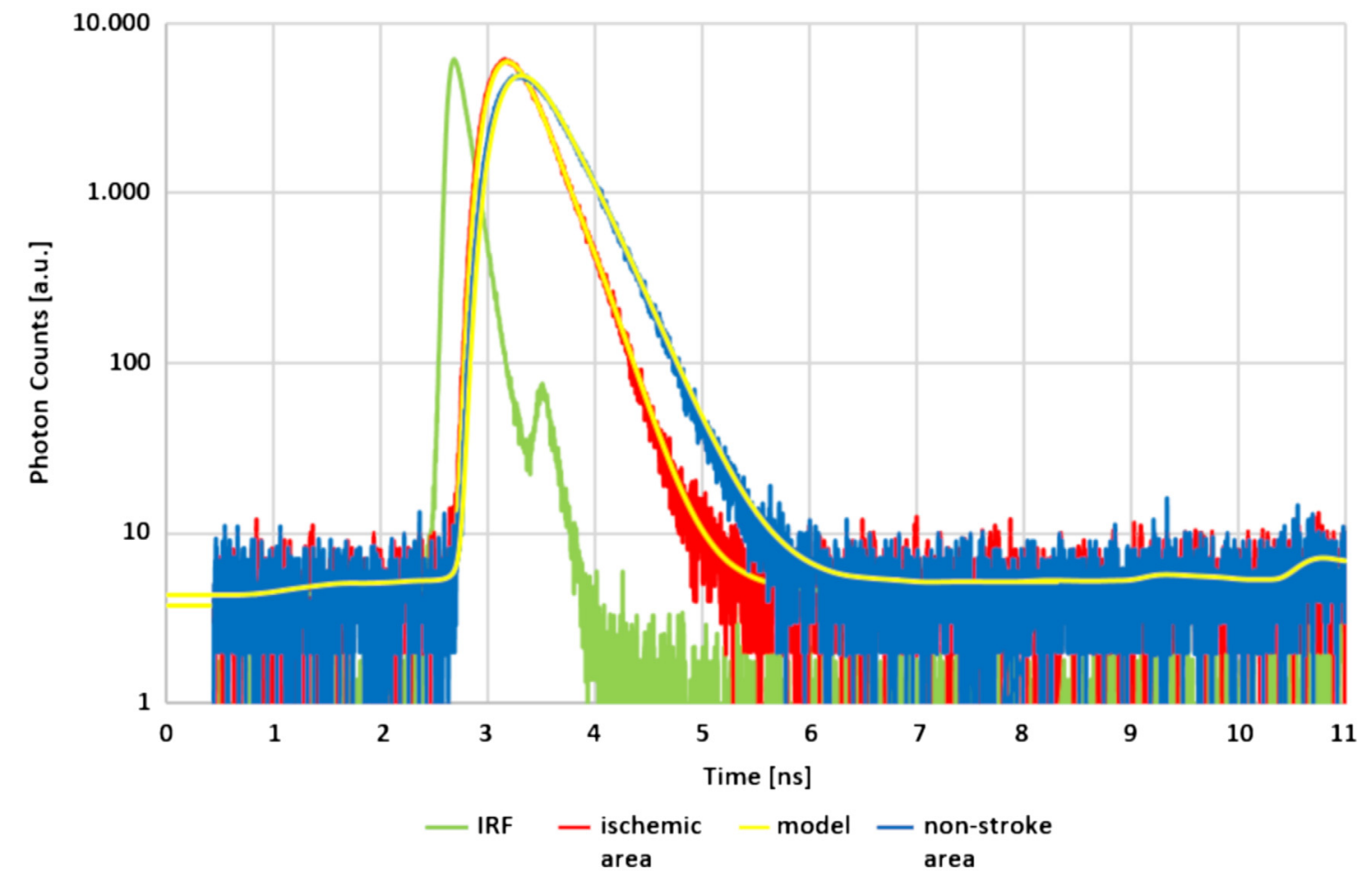

Fig. 3 Example of IRF (green line) and of distribution of photon time of flight (DTOF) at $690 \mathrm{~nm}$ from a position within the nonstroke contralateral region (source-detector position $\mathrm{F} 4$ to $\mathrm{F} 6$, blue line) and from a position within the ischemic area (source-detector position F3 to F5, red line) (data from patient \#20). In yellow best fits with the model of photon diffusion are also shown. 


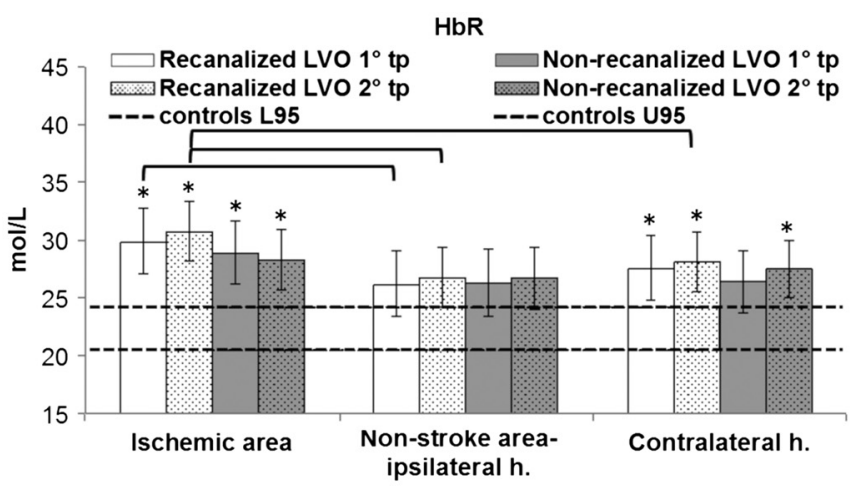

(a)

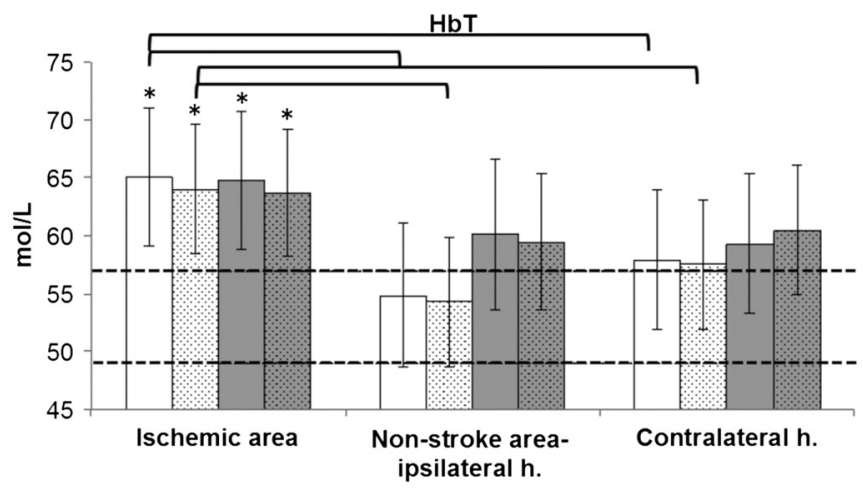

(c)

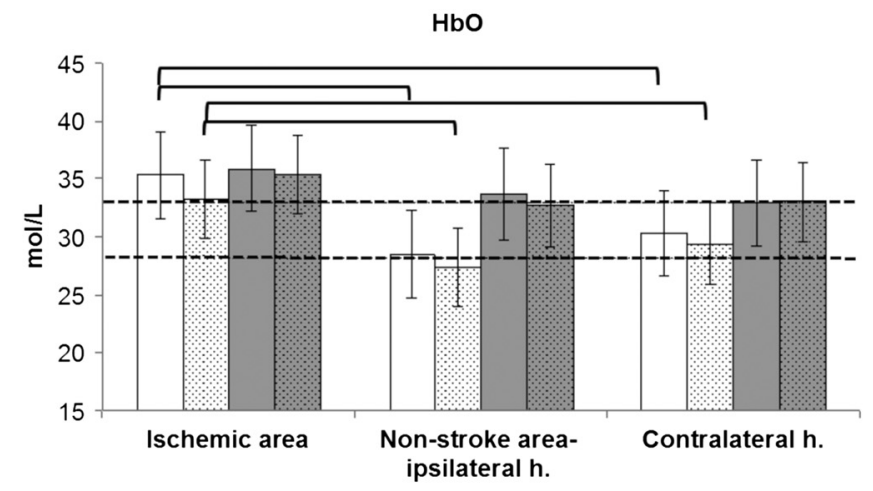

(b)

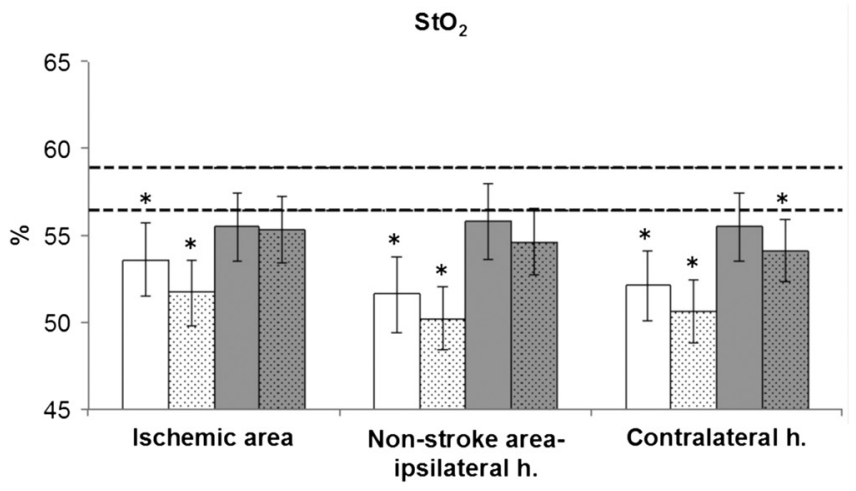

(d)

Fig. 4 Cerebral hemoglobin species and $\mathrm{StO}_{2}$ in $\mathrm{LVO}$ ischemic stroke patients and controls. Bar plots of cerebral hemoglobin species $(\mathrm{mol} / \mathrm{L})$ and $\mathrm{StO}_{2}(\%)$ in different brain areas, in LVO recanalized and nonrecanalized patients at first and second time points (tp). Error bars represent confidence intervals at $95 \%$. The range of normal values (confidence intervals at $95 \%$ ) from the control cohort of healthy subjects is shown as horizontal dashed lines. ${ }^{*} p<0.05$ in the statistical comparison with controls. Brackets indicate $p<0.05$ in the statistical comparison between areas and between time points.

compared with controls (Fig. 4 and Table 3); higher concentration of $\mathrm{HbR}(p=0.01), \mathrm{HbO}\left(p=3.6 \times 10^{-3}\right)$ and $\mathrm{HbT}$ $\left(p=2.0 \times 10^{-3}\right)$ compared with the ipsilateral nonstroke area; higher concentration of $\mathrm{HbO}(p=0.049), \mathrm{HbT}(p=0.03)$ compared with the contralateral hemisphere (Table 3). Measurements at the second time point were obtained at a mean interval of $84 \pm$ $25 \mathrm{~h}$ from onset. Compared with controls, recanalized patients had a pattern of hemoglobin species and $\mathrm{StO}_{2}$ similar to the first time point (Fig. 4 and Table 3). In recanalized LVO patients with available measurements at both time points, the concentration of hemoglobin species and $\mathrm{StO}_{2}$ in ischemic area and nonstroke areas of ipsilateral and contralateral hemisphere was comparable between first and second time points (Table 3).

Within $24 \mathrm{~h}$ from symptoms onset, nonrecanalized LVO patients had increased $\mathrm{HbR}$ in the ipsilateral hemisphere and increased $\mathrm{HbR}$ and $\mathrm{HbT}$ in the ischemic area compared with controls (respectively, HbR $p=5.9 \times 10^{-3}$; HbR $p=4.9 \times 10^{-3}$, HbT $p=0.03$ ) (Fig. 4 and Table 4). Measurements at the second time point were obtained at a mean interval of $86 \pm 25 \mathrm{~h}$ from onset. Compared with controls, nonrecanalized LVO patients had a pattern of hemoglobin species and $\mathrm{StO}_{2}$ similar to the first time point except that contralateral hemisphere had increased $\mathrm{HbR}$ and decreased $\mathrm{StO}_{2}\left(\mathrm{HbR} p=0.02, \mathrm{StO}_{2} p=0.03\right)$ (Fig. 4 and Table 4). In nonrecanalized LVO patients with available measurements at both time points, the concentration of hemoglobin species and $\mathrm{StO}_{2}$ in ischemic area and nonstroke areas of ipsilateral and contralateral hemisphere were comparable between first and second time points (Table 4).

At both time points, the ipsilateral hemisphere of recanalized LVO patients had lower mean $\mathrm{StO}_{2}$ compared with nonrecanalized LVO patients (respectively, $p=0.016, p=0.011$ ) and comparable $\mathrm{HbR}, \mathrm{HbO}$, and $\mathrm{HbT}$ (Tables 3 and 4).

Recanalized LVO patients with good functional outcome, defined as modified Rankin scale $(\mathrm{mRS}) \leq 3$ after 3 months, had a significantly higher $\mathrm{StO}_{2}$ in the ipsilateral hemisphere [first time point $53.7 \%( \pm 2.8)$ versus $46.1 \% \quad( \pm 3.7)$, $p=0.012$; second time point $51.9 \%( \pm 3.1)$ versus $47.7 \%$ $( \pm 4.2), p=0.042]$. Improvement of stroke severity, defined as a positive difference between baseline and discharge NIHSS, was associated with higher $\mathrm{StO}_{2}$ in recanalized $\mathrm{LVO}$ patients in the ipsilateral hemisphere [first time point $53.7 \%( \pm 2.8)$ versus $46.1 \%( \pm 3.7), p=0.012$; second time point $51.7 \%( \pm 3.0)$ versus $44.6 \%( \pm 3.7), p=0.006]$.

Patients with LACS stroke had similar concentration of hemoglobin species and $\mathrm{StO}_{2}$ values of both hemispheres compared with controls at both time points (Table 2).

\section{Discussion}

The present study shows that TD-NIRS is able to detect significant differences in hemoglobin species in LVO stroke compared with controls and according to recanalization status. TD-NIRS measurement is feasible in the real-life scenario of the stroke unit even on patients with severe functional impairment. 
Table 2 Estimated mean concentration of hemoglobin species and $\mathrm{StO}_{2}$ values in controls, LACS and LVO stroke patients according to linear mixed model.

\begin{tabular}{|c|c|c|c|c|c|}
\hline & $N$ & $\mathrm{HbR}^{\mathrm{a}}$ & $\mathrm{HbO}^{\mathrm{a}}$ & $\mathrm{HbT}^{\mathrm{a}}$ & $\mathrm{StO}_{2}{ }^{\mathrm{a}}$ \\
\hline Controls $^{b}$ & 35 & $22.3(0.9)$ & $30.5(1.2)$ & $52.9(2.0)$ & $57.6(0.7)$ \\
\hline \multicolumn{6}{|l|}{ Lacs } \\
\hline \multicolumn{6}{|l|}{ Ipsilateral hemisphere } \\
\hline First time point & 3 & $23.5(2.5)$ & $29.4(3.4)$ & $52.9(5.4)$ & $55.0(1.9)$ \\
\hline Second time point & 5 & $24.1(2.4)$ & $30.1(3.1)$ & $54.3(5.1)$ & $55.4(1.7)$ \\
\hline \multicolumn{6}{|c|}{ Contralateral hemisphere } \\
\hline First time point & 3 & $23.9(2.6)$ & $30.3(3.6)$ & $54.2(5.7)$ & $55.8(1.9)$ \\
\hline Second time point & 5 & $22.6(2.4)$ & $28.1(3.2)$ & $50.7(5.3)$ & $55.2(1.7)$ \\
\hline \multicolumn{6}{|l|}{ All LVO stroke patients } \\
\hline \multicolumn{6}{|l|}{ Ipsilateral hemisphere } \\
\hline First time point & 21 & $28.1(0.9)^{\mathrm{c}}$ & $33.5(1.3)$ & $61.5(2.0)$ & $54.0(0.7)^{\mathrm{c}}$ \\
\hline Second time point & 36 & $28.2(0.9)^{c}$ & $32.3(1.2)$ & $60.5(1.9)$ & $53.0(0.7)^{\mathrm{c}}$ \\
\hline \multicolumn{6}{|c|}{ Contralateral hemisphere } \\
\hline First time point & 21 & $27.0(0.9)^{\mathrm{c}}$ & $31.7(1.3)$ & $58.6(2.0)$ & $53.8(0.7)^{\mathrm{c}}$ \\
\hline Second time point & 36 & $27.8(0.9)^{\mathrm{c}}$ & $31.2(1.2)$ & $59.0(1.9)$ & $52.4(0.7)^{\mathrm{c}}$ \\
\hline
\end{tabular}

${ }^{\mathrm{a} C}$ Columns $\mathrm{HbR}, \mathrm{HbO}, \mathrm{HbT}, \mathrm{StO}_{2}$ represent mean (standard error); measure unit for $\mathrm{HbR}, \mathrm{HbO}, \mathrm{HbT}: \mathrm{mol} / \mathrm{L}$; measure unit for $\mathrm{StO} \mathrm{O}_{2}=\%$.

${ }^{b}$ Control values stem from a subgroup of Ref. 10.

${ }^{c} p$-value $<0.05$ in the statistical comparison with controls. $N=$ number of valid measurements for statistical analysis. LACS, lacunar anterior circulation syndrome; LVO, large vessel occlusion.

Table 3 Estimated mean concentration of hemoglobin species and $\mathrm{StO}_{2}$ values in controls and recanalized LVO stroke patients, according to linear mixed model.

\begin{tabular}{|c|c|c|c|c|c|}
\hline & $N$ & $\mathrm{HbR}^{\mathrm{a}}$ & $\mathrm{HbO}^{\mathrm{a}}$ & $\mathrm{HbT}^{\mathrm{a}}$ & $\mathrm{StO}_{2}{ }^{\mathrm{a}}$ \\
\hline Controls $^{b}$ & 35 & $22.3(0.9)$ & $30.5(1.2)$ & $52.9(2.0)$ & $57.6(0.7)$ \\
\hline \multicolumn{6}{|l|}{ Recanalized LVO } \\
\hline \multicolumn{6}{|l|}{ Ipsilateral hemisphere } \\
\hline First time point & 10 & $28.1(1.3)^{c}$ & $31.8(1.8)$ & $59.9(2.9)$ & $52.4(1.0)^{\mathrm{C}}$ \\
\hline Second time point & 18 & $28.8(1.2)^{\mathrm{c}}$ & $30.3(1.6)$ & $59.1(2.7)$ & $51.0(0.9)^{\mathrm{c}}$ \\
\hline \multicolumn{6}{|l|}{ Ischemic area } \\
\hline First time point & 10 & $29.9(1.4)^{\mathrm{c}}$ & $35.3(1.9)$ & $65.1(3.1)^{\mathrm{c}}$ & $53.6(1.0)^{\mathrm{c}}$ \\
\hline Second time point & 18 & $30.8(1.3)^{\mathrm{c}}$ & $33.2(1.7)$ & $64.0(2.8)^{\mathrm{c}}$ & $51.7(0.9)^{\mathrm{c}}$ \\
\hline \multicolumn{6}{|l|}{ Nonstroke area } \\
\hline First time point & 9 & $26.2(1.4)$ & $28.5(1.9)$ & $54.8(3.1)$ & $51.6(1.0)^{\mathrm{c}}$ \\
\hline Second time point & 18 & $26.8(1.3)$ & $27.4(1.7)$ & $54.3(2.8)$ & $50.2(0.9)^{\mathrm{c}}$ \\
\hline \multicolumn{6}{|c|}{ Contralateral hemisphere } \\
\hline First time point & 10 & $27.6(1.4)^{\mathrm{c}}$ & $30.3(1.9)$ & $57.9(3.1)$ & $52.1(1.0)^{\mathrm{C}}$ \\
\hline Second time point & 18 & $28.1(1.3)^{\mathrm{c}}$ & $29.4(1.7)$ & $57.5(2.8)$ & $50.6(0.9)^{\mathrm{c}}$ \\
\hline
\end{tabular}

${ }^{\mathrm{a} C}$ Columns $\mathrm{HbR}, \mathrm{HbO}, \mathrm{HbT}, \mathrm{StO}_{2}$ represent mean (standard error); measure unit for $\mathrm{HbR}, \mathrm{HbO}, \mathrm{HbT}$ : mol/L; measure unit for $\mathrm{StO}{ }_{2}=\%$.

${ }^{\mathrm{b} C o n t r o l}$ values stem from a subgroup of Ref. 10.

${ }^{c} p$-value $<0.05$ in the statistical comparison with controls. $N=$ number of valid measurements for statistical analysis. LACS, lacunar anterior circulation syndrome; LVO, large vessel occlusion. 
Table 4 Estimated mean concentration of hemoglobin species and $\mathrm{StO}_{2}$ values in controls and nonrecanalized LVO stroke patients, according to linear mixed model.

\begin{tabular}{lccccc} 
& $N$ & $\mathrm{HbR}^{\mathrm{a}}$ & $\mathrm{HbO}^{\mathrm{a}}$ & $\mathrm{HbT}^{\mathrm{a}}$ & $\mathrm{StO}_{2}{ }^{\mathrm{a}}$ \\
\hline Controls $^{\mathrm{b}}$ & 35 & $22.3(0.9)$ & $30.5(1.2)$ & $52.9(2.0)$
\end{tabular}

Nonrecanalized LVO

Ipsilateral hemisphere

First time point

Second time point

Second time point

Nonstroke area

First time point

Second time point

Contralateral hemisphere

First time point

Second time point

$\begin{array}{ll}7 & 26.3(1.5) \\ 12 & 26.7(1.4)\end{array}$

12

11

18

$26.7(1.4)$

$26.4(1.4)$

$27.5(1.3)^{\mathrm{c}}$ $28.0(1.3)^{\mathrm{c}}$

$27.7(1.2)^{\mathrm{c}}$

$28.9(1.4)^{\mathrm{c}}$

$28.3(1.3)^{\mathrm{c}}$
$35.2(1.8)$

$34.3(1.6)$

$35.9(1.9)$

$35.4(1.7)$

$33.8(2.1)$

$32.7(1.8)$

$32.9(1.9)$

$63.0(11.3)$

$33.0(1.7)$

$62.0(2.7)$

$68.4(9.1)^{\mathrm{c}}$

$63.7(10.7)^{\mathrm{c}}$

$65.6(11.6)$

$59.1(14.9)$

$55.7(1.0)$

$54.9(0.9)$

$55.5(1.0)$

$55.3(0.9)$

$55.8(1.1)$

$54.6(1.0)$

${ }^{\mathrm{a} C}$ Columns $\mathrm{HbR}, \mathrm{HbO}, \mathrm{HbT}, \mathrm{StO}_{2}$ represent mean (standard error); measure unit for $\mathrm{HbR}, \mathrm{HbO}, \mathrm{HbT}: \mathrm{mol}^{\mathrm{L}}$; measure unit for $\mathrm{StO}{ }_{2}=\%$.

${ }^{b}$ Control values stem from a subgroup of Ref. 10.

${ }^{c} p$-value $<0.05$ in the statistical comparison with controls. $N=$ number of valid measurements for statistical analysis. LACS, lacunar anterior circulation syndrome; LVO, large vessel occlusion.

TD-NIRS is an advanced noninvasive optical technique to study real-time oxygenation of cerebral outer layers at the bedside of the patient. ${ }^{6}$ Previous NIRS studies of ischemic stroke were performed mainly with the CW-NIRS technique that measures relative changes of $\mathrm{HbR}, \mathrm{HbO}, \mathrm{HbT}$, and $\mathrm{StO}_{2}$ compared with a baseline. ${ }^{11-16}$ TD-NIRS enables better separation of the signal origin as well as absolute concentration measurements. Quantitative measurements would allow establishing the normative values, to compare normal and pathological conditions and monitor the evolution of the disease. In addition, previous NIRS studies mainly recorded only from the forehead, ${ }^{11-16}$ regardless of the site of ischemic injury. A recent study with FD-NIRS on five acute stroke patients performed measurement on multiple sites selected according to imaginary lines drawn through surface anatomical landmarks. ${ }^{17}$

In our study, we used fiducial markers to categorize the brain region below each TD-NIRS probe as ischemic or nonstroke areas. Patients with LACS acute ischemic stroke had TDNIRS values comparable with controls. Considering the physical properties of light propagation in the tissue, we quite expected that TD-NIRS would be of limited value to pick-up changes in absorption and scattering of deep cerebral tissue and the derived hemoglobin species concentrations. In addition, the superficial venous compartment is by-passed because the venous drainage of the deep cerebral tissue belongs to the deep cerebral venous system. ${ }^{23}$ The physical limits of light propagation typical of TD-NIRS (and of the NIRS technique in general) explain the result of LACS stroke that may serve as internal negative control.
Overall, patients with LVO acute ischemic stroke, regardless of recanalization status, had a significantly higher concentration of $\mathrm{HbR}$ and significantly decreased $\mathrm{StO}_{2}$ values in ipsilateral hemisphere compared with control subjects. In addition, we found different TD-NIRS patterns according to the recanalization status of the patients and the use of fiducial markers allowed us to separate TD-NIRS measurements obtained from ischemic areas and nonstroke areas. Indeed, ischemic and nonstroke areas of acute ischemic stroke patients featured significantly different TD-NIRS values compared with controls. Recanalized patients as compared with controls had lower $\mathrm{StO}_{2}$ in ischemic area and nonstroke areas of both hemispheres. Furthermore, the ischemic area had a higher concentration of $\mathrm{HbR}$ and HbT. The changes in hemoglobin species and $\mathrm{StO}_{2}$ are certainly due to a complex process resulting from the interaction of several factors. A potential role played by respiratory insufficiency in reducing $\mathrm{StO}_{2}$ was ruled out by normal peripheral arterial pulse oxygen saturation $\left(\mathrm{SpO}_{2}>94 \%\right)$ during TD-NIRS measurements; furthermore, we did not find any correlation between $\mathrm{SpO}_{2}$ and TD-NIRS values. Considering that recanalized patients feature restoration of arterial blood flow within a metabolically challenged area, the observed pattern might reflect the activation of global hemodynamic and metabolic compensation to the ischemic injury (vasodilation resulting in increased $\mathrm{HbT},{ }^{24}$ oxygen extraction resulting in reduced $\mathrm{StO}_{2}{ }^{25}$ ). The increase in lactate and local acidosis ${ }^{26,27}$ might act as a chemical vasodilatory stimulus in the ischemic area. On the other hand, the lower $\mathrm{StO}_{2}$ values in ipsilateral and contralateral hemisphere compared with controls could be a marker of increased oxygen extraction due to 
the increased metabolic activity of the rescued brain tissue, increased glutamate in the peri-infarct area ${ }^{28}$ along with cortical activation of nonstroke areas. In addition, the increase in oxygen extraction in the ischemic area might also be due to the Bohr's effect of local acidosis with a shift of the $\mathrm{O}_{2}-\mathrm{Hb}$ dissociation curve toward right. ${ }^{29}$ Differences in vascular resistance in the microcirculation between the ischemic area and the ipsilateral nonstroke area might cause a preferential distribution of flow to the ischemic area, which would explain the higher concentration of $\mathrm{HbO}$ and $\mathrm{HbT}$ compared with the ipsilateral nonstroke area. It is known that ischemic stroke may induce widespread cerebral hemodynamic changes and activation of plasticity mechanisms. ${ }^{3-5}$ Arterial and venous collateral pathways and hemodynamic vasodilation compensatory mechanisms may act also on noninfarcted brain areas. Functional MRI studies demonstrated widespread cortical activation in the acute phase in areas remote from ischemic lesions, suggestive of neuronal plasticity. ${ }^{30-33}$

Nonrecanalized patients had higher $\mathrm{HbR}$ and $\mathrm{HbT}$ but similar $\mathrm{StO}_{2}$ values in the ischemic area compared with controls. Supposedly, the ischemic area of nonrecanalized patients might feature residual blood flow from leptomeningeal arterial and venous collaterals, altered venous drainage and reduced oxygen consumption because of absent metabolic activity of the infarcted tissue. The observed higher concentrations of $\mathrm{HbR}$ and HbT may also reflect blood stagnation through a deranged ischemic microcirculation. Interestingly, recanalized patients had lower $\mathrm{StO}_{2}$ values compared with nonrecanalized patients suggesting that $\mathrm{StO}_{2}$ could be a potential surrogate marker of metabolically active tissue.

We acknowledge that our study has a number of limitations among which the use of a prototype of TD-NIRS in a single center. In addition, the use of a homogenous model of photons' diffusion instead of a two-layer algorithm is a possible limitation of our study. At present, the analysis of TD-NIRS data with a two-layer model is a time-consuming process that requires higher computational resources than a simple homogeneous model,${ }^{34}$ which is being inadequate for a prompt clinical decision setting such as the acute ischemic stroke. However, future studies might address whether the use of a two-layer model will yield comparable results. The sample size of our study, albeit being among the largest in NIRS studies in stroke so far, is still limited. Indeed, owing to the small sample size, the analysis to examine the association of neurological outcome with hemoglobin species and $\mathrm{StO}_{2}$ was merely explorative and as such is not appropriate to derive any conclusion. In addition, due to the real life setting of the study, advanced stroke imaging was not a requirement, thus we cannot exclude that this could have limited the ability to detect potential changes in the extension of the ischemic stroke area between the first and second time points. On the other hand, a major change in extension of the ischemic area would have an effect on clinical severity, but we found a nonsignificant change of NIHSS between the two time points. Nevertheless, the results of the present study need to be interpreted with caution and certainly have to be confirmed on a larger sample. The absence of a concomitant measurement of local cerebral blood flow is prevented to enrich TD-NIRS data and derive a more accurate insight on the metabolism of the probed brain areas. We are planning to implement the concomitant use of diffuse correlation spectroscopy (DCS $)^{35}$ with the development of hybrid systems. Finally, ischemic brain tissue may undergo structural changes among the occurrence of cytotoxic and vasogenic edema. In cytotoxic and vasogenic edema, water is redistributed, respectively, from the extracellular to intracellular space and from vascular blood to extracellular space. Brain edema develops in a closed system such that an increase in local brain tissue volume due to edema is partially compensated by a reduction in the cerebrospinal fluid component resulting in a change of compartimentalization of water. In addition, the water absorption in the spectral range of interest is rather low. On this ground, we do not expect major changes in the calculation of the absorption coefficient. Furthermore, the fitting process and the hemoglobin estimates accounted for possible modifications of scattering, which is mainly due to the refractive index mismatch of the different tissue components.

Measurements of peripheral blood hemoglobin, arterial blood pressure, and $\mathrm{SpO}_{2}$ during the recording sessions minimized the risk of misinterpretation related to imbalance of systemic homeostatic parameters.

In summary, in this pivotal study we found significant differences of TD-NIRS values between patients and controls and among patients according to recanalization status. The ischemic area of both recanalized and nonrecanalized patients featured increased $\mathrm{HbR}$ and $\mathrm{HbT}$ that might reflect the hemodynamic compensation to the ischemic injury but only recanalized patient had reduced $\mathrm{StO}_{2}$, a finding that might reflect the metabolic activity of the rescued brain tissue. The results obtained from this cohort of acute stroke patients suggest that $\mathrm{StO}_{2}$ could serve as surrogate functional marker metabolic activity of rescued brain tissue and might be an additional parameter to account for in the prognostic process of acute stroke patients. Correlation of TD-NIRS with other techniques such as DCS may better inform on tissue viability and blood flow.

\section{Disclosures}

The authors have no conflicts of interest to declare.

\section{Acknowledgments}

G.G. and L.R. made substantial contribution to the study conception and design and contributed to the statistical analyses of data, to their interpretation and to the drafting of the manuscript. G.G., M.Z., R.R., B.G., D.C., L.S., A.T. contributed to the acquisition of data. M.Z., R.R., D.C., L.S., A.T. contributed to the analysis of TD-NIRS data. All authors contributed to critical comments and revision of the paper. This study was not funded by any specific project grant.

\section{References}

1. L. Østergaard et al., "The role of the cerebral capillaries in acute ischemic stroke: the extended penumbra model," J. Cereb. Blood Flow Metab. 33, 635-648 (2013).

2. J. H. Zhang et al., "The vascular neural network-a new paradigm in stroke pathophysiology," Nat. Rev. Neurol. 8, 711-716 (2012).

3. L. Ruan et al., "Metabolite changes in the ipsilateral and contralateral cerebral hemispheres in rats with middle cerebral artery occlusion," Neural Regener. Res. 12, 931-937 (2017).

4. G. Rubin et al., "Remote effects of acute ischemic stroke: a xenon CT cerebral blood flow study," Cerebrovasc. Dis. 10, 221-228 (2000).

5. J. A. Dobkin et al., "Evidence for transhemispheric diaschisis in unilateral stroke," Arch. Neurol. 46, 1333-1336 (1989).

6. A. Torricelli et al., "Time domain functional NIRS imaging for human brain mapping," Neuroimage 85, 28-50 (2014).

7. H. Obrig, "NIRS in clinical neurology-a 'promising' tool?" Neuroimage 85, 535-546 (2014). 
8. F. Scholkmann et al., "A review on continuous wave functional nearinfrared spectroscopy and imaging instrumentation and methodology," Neuroimage 85, 6-27 (2014).

9. A. Giusto et al., "Monitoring absorption changes in a layered diffusive medium by white-light time-resolved reflectance spectroscopy," IEEE Trans. Instrum. Meas. 59, 1925-1932 (2010).

10. G. Giacalone et al., "Cerebral time domain-NIRS: reproducibility analysis, optical properties, hemoglobin species and tissue oxygen saturation in a cohort of adult subjects," Biomed. Opt. Express 8, 4987-5000 (2017).

11. A. C. Flint et al., "Detection of anterior circulation large artery occlusion in ischemic stroke using noninvasive cerebral oximetry," Stroke 49 , 458-460 (2018).

12. T. Ritzenthaler et al., "Cerebral near-infrared spectroscopy: a potential approach for thrombectomy monitoring," Stroke 48, 3390-3392 (2017).

13. T. Ritzenthaler et al., "Usefulness of near-infrared spectroscopy in thrombectomy monitoring," J. Clin. Monit. Comput. 29, 585-589 (2015).

14. C. Hametner et al., "Noninvasive cerebral oximetry during endovascular therapy for acute ischemic stroke: an observational study," J. Cereb. Blood Flow Metab. 35, 1722-1728 (2015).

15. F. Pizza et al., "Cerebral hemodynamic changes in stroke during sleep-disordered breathing," Stroke 43, 1951-1953 (2012).

16. M. S. Damian and R. Schlosser, "Bilateral near infrared spectroscopy in space-occupying middle cerebral artery stroke," Neurocrit. Care 6, 165-173 (2007).

17. R. Delgado-Mederos et al., "Transcranial diffuse optical assessment of the microvascular reperfusion after thrombolysis for acute ischemic stroke," Biomed. Opt. Express 9, 1262-1271 (2018).

18. F. Moreau et al., "Near-infrared measurements of brain oxygenation in stroke," Neurophotonics 3, 031403 (2016).

19. T. Durduran et al., "Transcranial optical monitoring of cerebrovascular hemodynamics in acute stroke patients," Opt. Express 17, 3884-3902 (2009).

20. N. Asdaghi et al., "Oxfordshire community stroke project classification poorly differentiates small cortical and subcortical infarcts," Stroke 42, 2143-2148 (2011).

21. P. A. Barber et al., "Validity and reliability of a quantitative computed tomography score in predicting outcome of hyperacute stroke before thrombolytic therapy," Lancet 355, 1670-1674 (2000).

22. L. Tatu et al., "Arterial territories of the human brain," Front. Neurol. Neurosci. 30, 99-110 (2012).

23. T. Kilic and A. Akakin, "Anatomy of cerebral veins and sinuses," Front. Neurol. Neurosci. 23, 4-15 (2008).

24. G. Strangman et al., "A quantitative comparison of simultaneous BOLD fMRI and NIRS recordings during functional brain activation," Neuroimage 17, 719-731 (2002).

25. G. Naulaers et al., "Use of tissue oxygenation index and fractional tissue oxygen extraction as non-invasive parameters for cerebral oxygenation," Neonatology 92, 120-126 (2007).

26. J. Mohr et al., Pathophysiology, Diagnosis and Management, 5th edn., Elsevier Saunders, Philadelphia (2011).

27. R. Leigh et al., "Imaging the physiological evolution of the ischemic penumbra in acute ischemic stroke," J. Cereb. Blood Flow Metab. 38, 1-17 (2017).

28. A. Bivard et al., "Spectroscopy of reperfused tissue after stroke reveals heightened metabolism in patients with good clinical outcomes," J. Cereb. Blood Flow Metab. 34, 1944-1950 (2014).

29. J. A. Collins et al., "Relating oxygen partial pressure, saturation and content: the haemoglobin-oxygen dissociation curve," Breathe 11, 194-201 (2015).

30. N. S. Ward, "Neural correlates of motor recovery after stroke: a longitudinal fMRI study," Brain 126, 2476-2496 (2003).

31. A. K. Rehme et al., "Dynamic causal modeling of cortical activity from the acute to the chronic stage after stroke," Neuroimage 55, 1147-1158 (2011).

32. A. K. Rehme et al., "The role of the contralesional motor cortex for motor recovery in the early days after stroke assessed with longitudinal fMRI," Cereb. Cortex 21, 756-768 (2011).
33. T. Askim et al., "Motor network changes associated with successful motor skill relearning after acute ischemic stroke: a longitudinal functional magnetic resonance imaging study," Neurorehabil. Neural Repair 23, 295-304 (2009).

34. F. Martelli et al., Light Propagation through Biological Tissue and other Diffusive Media: Theory, Solutions, and Software, SPIE Press, Bellingham, Washington (2009).

35. T. Durduran and A. G. Yodh, "Diffuse correlation spectroscopy for non-invasive, micro-vascular cerebral blood flow measurement," Neuroimage 85, 51-63 (2014).

Giacomo Giacalone received his degree in medicine, the specialty in neurology, and his PhD in experimental neurology from San Raffaele University in Milan in 2008, 2014, and 2018, respectively. He works at the Stroke Unit of the Neurology Department of San Raffaele Hospital in Milan as clinician and as researcher. In 2015, he promoted the set up of a clinical research laboratory for the application of advanced optical device in ischemic stroke and other neurological diseases.

Marta Zanoletti received her MS degree in engineering physics from Politecnico di Milano in 2015. In 2016, she enrolled in the PhD program at the Physics Department of Politecnico di Milano. Her research focuses on the study and application of time domain infrared spectroscopy technique to the medical field.

Rebecca $\mathrm{Re}$ received her MS degree in physical engineering and her PhD in physics from Politecnico di Milano, Italy, in 2008 and 2012, respectively. She is a research fellow at the Department of Physics, Politecnico di Milano. Her research interests focus on photon migration in diffusive media, the development of instruments for timedomain functional near-infrared spectroscopy (TD fNIRS), and their application in clinical settings.

Bruno Germinario obtained his degree in medicine at San Raffaele University in 2017. For his degree thesis, he worked on the application of TD-NIRS in acute ischemic stroke patients.

Davide Contini received his MS degree in electronic engineering and his PhD in physics from Politecnico di Milano in 2004 and 2007, respectively. He is an associate professor in the Department of Physics, Politecnico di Milano, Italy. He is the author of more than 150 papers in international peer-reviewed journals and conference proceedings. His research activity is focused on time-resolved spectroscopy of highly diffusive media for applications in biology and medicine.

Lorenzo Spinelli received his MS and PhD degrees in physics from the University of Milan, Italy, in 1994 and 1999, respectively. Since 1999, he has been a postdoc in the Department of Physics at the University of Milan. In 2001, he became a researcher for Italian Research National Council at Institute of Photonics and Nanotechnologies. His research interests are devoted to the study of photon migration in turbid media for optical biopsy and imaging.

Alessandro Torricelli is a full professor at the Department of Physics, Politecnico di Milano (Italy). He received his MS degree in electronic engineering from Politecnico di Milano in 1994, and his $\mathrm{PhD}$ in physics from Politecnico di Torino in 1999. He is the author of more than 100 papers on international peer-reviewed journals. His current research interests include photon migration in diffusive media, functional near-infrared spectroscopy, and noninvasive diffuse spectroscopy with time domain systems.

Luisa Roveri has been a neurologist at the Neurology Department of San Raffaele Hospital in Milan since 1996. Since 2014, she has been the head of Stroke Unit at San Raffaele Hospital in Milan. She has been the principal investigator in several clinical trials on stroke patients. In 2015, she set up a clinical research laboratory for the application of advanced optical devices in ischemic stroke and served as a director of study for the PhD in experimental neurology. 